Real Analysis Exchange

Vol. 26(1), 2000/2001, pp. 485-488

Giovanni Alberti*, Dipartimento di Matematica, Università di Pisa, v. Buonarroti 2, 56127 Pisa, Italy. e-mail: alberti@ruffini.dm.unipi.it Marianna Csörnyei† Department of Mathematics, University College London, Gower Street, London WC1E 6BT, UK. e-mail: mari@math.ucl.ac.uk Miklós Laczkovich Kecskeméti u. 12, H-1053, Hungary. e-mail: laczko@renyi.hu David Preiss, Department of Mathematics, University College London, Gower Street, London WC1E 6BT, UK. e-mail: dp@math.ucl.ac.uk

\title{
DENJOY-YOUNG-SAKS THEOREM FOR APPROXIMATE DERIVATIVES REVISITED
}

\begin{abstract}
We note that the restriction of any measurable mapping $f: \mathbb{R} \rightarrow \mathbb{R}^{n}$ to the set of points at which it possesses a finite approximate derived number maps Lebesgue null sets to sets of zero linear measure. As a corollary we deduce an optimal version of Denjoy-Young-Saks theorem for approximate derivatives valid up to exceptional sets of zero linear measure in the graph.
\end{abstract}

Denjoy-Young-Saks Theorems are results saying that except for $x$ belonging to a small set the Dini (extreme unilateral) derivatives satisfy certain non-trivial relations. For example, the Dini derivatives of an arbitrary function $f: \mathbb{R} \rightarrow \mathbb{R}$ satisfy one of the following four relations at almost every point $x$ :

(i) $\bar{f}^{+}(x)=\bar{f}^{-}(x)=\underline{f}^{+}(x)=\underline{f}^{-}(x)$ is finite.

(ii) $\bar{f}^{+}(x)=\underline{f}^{-}(x)$ is finite, $\underline{f}^{+}(x)=-\infty, \bar{f}^{-}(x)=\infty$.

Key Words: Denjoy-Young-Saks theorem, approximate Dini derivatives, Lusin's property $(\mathrm{N})$

Mathematical Reviews subject classification: 26A24, 28A75

Received by the editors October 12, 2000

* Supported by GNAFA

${ }^{\dagger}$ Supported by the Hungarian National Foundation for Scientific Research, grant \# F029768.

${ }^{\ddagger}$ Supported by the Hungarian National Foundation for Scientific Research, grant \# T032042 
(iii) $\underline{f}^{+}(x)=\bar{f}^{-}(x)$ is finite, $\bar{f}^{+}(x)=\infty, \underline{f}^{-}(x)=-\infty$.

(iv) $\bar{f}^{+}(x)=\bar{f}^{-}(x)=+\infty, \underline{f}^{+}(x)=\underline{f}^{-}(x)=-\infty$.

Similarly, the approximate Dini derivatives of any measurable function $f$ : $\mathbb{R} \rightarrow \mathbb{R}$ satisfy, at almost every point $x$, one of the two relations (i) and (iv).

For ordinary Dini derivatives, Theorems 9.4.2-9.4.4 in [1] contain not only the almost everywhere version of Denjoy-Young-Saks Theorem but also its version with exceptional sets of zero linear measure in the graph: In the latter case the complete list of relations is obtained by deleting the phrase "is finite" from (i)-(iv). However, for approximate Dini derivatives we are aware only of the almost everywhere version, which follows immediately from the DenjoyKhintchine Theorem [1, Theorem 9.10.1]). Here we intend to complete the picture by finding all relations among the approximate Dini derivatives valid up to sets of zero linear measure in the graph.

It is easy to see that the missing piece of information is related to a version of Lusin's property $(\mathrm{N})$. In [1, Theorem 9.9.1-9.9.7] one can find a beautiful argument showing that the restriction of any measurable function $f: \mathbb{R} \rightarrow \mathbb{R}$ to the set $E$ of points at which at least one approximate derived number is finite has Lusin's property (N), i.e. it maps Lebesgue null subsets of $E$ to Lebesgue null sets. Unfortunately, the argument is purely one-dimensional, while we need to know that the (vector-valued) mapping $x \mapsto(x, f(x))$ maps Lebesgue null subsets of $E$ to planar sets of linear (Hausdorff) measure zero. Our main observation says that this holds even for arbitrary mappings between Euclidean spaces. To formulate it, we denote by $\mathcal{L}^{k}$ and $\mathcal{H}^{k}$ the outer Lebesgue measure in $\mathbb{R}^{k}$ and the $k$-dimensional outer Hausdorff measure in $\mathbb{R}^{n}$, respectively, by $\alpha(k)$ the Lebesgue measure of the unit ball in $\mathbb{R}^{k}$, and by $B_{n}(x, r)$ the ball centered at $x \in \mathbb{R}^{n}$ with radius $r$.

Theorem 1. Let $F: \mathbb{R}^{k} \rightarrow \mathbb{R}^{n}$ be an arbitrary mapping and let $E \subset \mathbb{R}^{k}$ and $\varepsilon>0$ be such that for every $x \in E$ and $\delta>0$ there are arbitrarily small values of $r$ for which the set $B_{k}(x, \delta) \cap f^{-1}\left(B_{n}(F(x), r)\right)$ has inner Lebesgue measure at least $\varepsilon \alpha(k) r^{k}$. Then

$$
\mathcal{H}^{k}(F(E)) \leq \frac{N}{\varepsilon} \mathcal{L}^{k}(E),
$$

where $N$ is the constant in the $n$-dimensional version of Besicovitch's covering theorem.

Proof. Let us fix $\delta>0$ and an open set $A$ which contains $E$ and satisfies $\mathcal{L}^{k}(A) \leq \mathcal{L}^{k}(E)+\delta$, and consider the covering $\mathcal{F}$ of $F(E)$ given by all balls $B_{n}(F(x), r)$ with $x \in E$ and $0<r<\delta / 2$ taken so that the set $E(x, r)=$ $A \cap F^{-1}\left(B_{n}(F(x), r)\right)$ has inner Lebesgue measure at least $\varepsilon \alpha(k) r^{k}$. By the 
Besicovitch covering theorem, we can extract $N$ disjoint subfamilies $\mathcal{F}_{i}$ which still cover $F(E)$.

Fix $i$ for the time being. Since the balls $B=B_{n}(F(x), r)$ in $\mathcal{F}_{i}$ are disjoint, so are the corresponding sets $E(x, r)$ and therefore

$$
\begin{aligned}
\mathcal{L}^{k}(E)+\delta & \geq \mathcal{L}^{k}(A) \geq \sum \mathcal{L}^{k}(E(x, r)) \geq \sum \varepsilon \alpha(k) r^{k} \\
& =\varepsilon \sum_{B \in \mathcal{F}_{i}} \alpha(k) 2^{-k} \operatorname{diam}^{k}(B) .
\end{aligned}
$$

Then

$$
N\left(\mathcal{L}^{k}(E)+\delta\right) \geq \varepsilon \mathcal{H}_{\delta}^{k}(F(E))
$$

and the statement follows by letting $\delta \rightarrow 0$.

Corollary 2. Let $f: \mathbb{R} \rightarrow \mathbb{R}$ be a measurable function. Then there is a set $E \subset \mathbb{R}$ such that $\mathcal{H}^{1}\{(x, f(x)): x \in E\}=0$ and for every $x \in \mathbb{R} \backslash E$ one of the following two relations holds:

(i) $f$ has a finite approximate derivative at $x$.

(ii) All four approximate Dini derivatives of $f$ at $x$ are infinite.

Proof. The set $E$ of points at which none of the two relations holds is of Lebesgue measure zero by Denjoy-Young-Saks theorem for approximate derivatives, and, by [1, Theorem 9.9.1], there is a decomposition $E=\bigcup_{n=1}^{\infty} E_{n}$ such that $E_{n}$ satisfies the assumptions of our main theorem with $\varepsilon=1 / n$. So the graph of $f$ above $E$ has $\mathcal{H}^{1}$ measure zero.

Remark 3. Except for the obvious inequalities between lower and upper unilateral derivatives, the above relations fully describe the behavior of approximate Dini derivatives up to the required type of exceptional sets. To see this, denote by $E_{f}\left(\alpha_{1}, \alpha_{2}, \alpha_{3}, \alpha_{4}\right)$ the set of the points $x$ at which the approximate unilateral derivatives $\bar{f}_{a p p}^{+}, \underline{f}_{a p p}^{+}, \bar{f}_{a p p}^{-}, \underline{f}_{a p p}^{-}$are $\alpha_{1}, \alpha_{2}, \alpha_{3}, \alpha_{4}$, respectively. Then for every choice $\alpha_{1}, \alpha_{2}, \alpha_{3}, \alpha_{4} \in\{-\infty,+\infty\}$ such that $\alpha_{1} \geq \alpha_{2}$ and $\alpha_{3} \geq \alpha_{4}$ we may construct a continuous function $f$ for which the set $f\left(E_{f}\left(\alpha_{1}, \alpha_{2}, \alpha_{3}, \alpha_{4}\right)\right)$ has positive Lebesgue measure; consequently, the graph of $f$ above $E_{f}\left(\alpha_{1}, \alpha_{2}, \alpha_{3}, \alpha_{4}\right)$ has positive $\mathcal{H}^{1}$ measure.

Indeed, let $C$ denote the ternary Cantor set and let $f_{0}$ be the Cantor function on $[0,1]$. Then

$$
\mathcal{L}^{1}\left(E_{f_{0}}(\infty, \infty, \infty, \infty)\right)=1 .
$$

We fix a number $1 / 2<c<1$, define

$$
f_{1}(x)=f_{2}(x)=f_{3}(x)=f_{0}(x) \quad \text { for } x \in C
$$


and, whenever $[a, b]=\left[\ell / 3^{k},(\ell+1) / 3^{k}\right]$ is a contiguous interval of $C$,

$$
\begin{aligned}
& f_{1}(x)=f_{0}(x)-c^{k} \quad \text { for } x \in[a+(b-a) / 3, a+2(b-a) / 3] ; \\
& f_{2}(x)= \begin{cases}f_{0}(x) & x \in[a, a+(b-a) / 4], \\
f_{0}(x)-c^{k} & x \in[a+2(b-a) / 4, a+3(b-a) / 4] ;\end{cases} \\
& f_{3}(x)= \begin{cases}f_{0}(x)+c^{k} & x \in[a+(b-a) / 5, a+2(b-a) / 5], \\
f_{0}(x)-c^{k} & x \in[a+3(b-a) / 5, a+4(b-a) / 5]\end{cases}
\end{aligned}
$$

Finally, we extend the functions $f_{1}, f_{2}, f_{3}$ linearly to all intervals on which they have not been defined yet.

It is straightforward to check that the sets $f_{1}\left(E_{f_{1}}(-\infty,-\infty,+\infty,+\infty)\right)$, $f_{2}\left(E_{f_{2}}(+\infty,-\infty,+\infty,+\infty)\right)$ and $f_{3}\left(E_{f_{3}}(+\infty,-\infty,+\infty,-\infty)\right)$ have Lebesgue measure one. By replacing the functions $f_{i}(x)$ by $-f_{i}(x), f_{i}(-x)$ and $-f_{i}(-x)$ we obtain examples for all the other cases.

Incidentally, for $f_{0}, f_{2}$ and $f_{3}$ the approximate and ordinary Dini derivatives coincide, so these functions also form pertinent examples to the case of ordinary derivatives. Of course, this is impossible for $f_{1}$, which illustrates the case when both unilateral approximate derivatives are infinite and of opposite sign.

\section{References}

[1] S. Saks, Theory of the integral, Hefner Publishing Company, New York, 1937. 